\title{
Path Loss Model with Antenna Height Dependency under Indoor Stair Environment
}

\author{
Yu Yu, Yang Liu, Wen-Jun Lu, and Hong-Bo Zhu \\ Jiangsu Key Laboratory of Wireless Communications, College of Telecommunications and Information Engineering, \\ Nanjing University of Posts \& Telecommunications, Nanjing 210003, China \\ Correspondence should be addressed to Hong-Bo Zhu; zhb@njupt.edu.cn
}

Received 23 May 2014; Revised 18 June 2014; Accepted 6 July 2014; Published 22 July 2014

Academic Editor: Ahmad Safaai-Jazi

Copyright (C) $2014 \mathrm{Yu} \mathrm{Yu} \mathrm{et} \mathrm{al.} \mathrm{This} \mathrm{is} \mathrm{an} \mathrm{open} \mathrm{access} \mathrm{article} \mathrm{distributed} \mathrm{under} \mathrm{the} \mathrm{Creative} \mathrm{Commons} \mathrm{Attribution} \mathrm{License,} \mathrm{which}$ permits unrestricted use, distribution, and reproduction in any medium, provided the original work is properly cited.

\begin{abstract}
A novel, receiving antenna-height-dependent path loss model under indoor stair environment is presented. The effect of a crossbeam in the stairs is also considered. The proposed model can be applied to describe both of the line-of-sight (LOS) and the nonLOS (NLOS) cases. By using least square criterion, the parameters of proposed model are extracted. Finally, using the maximum likelihood estimation, the precision of the proposed model is evaluated by the standard deviation of shadowing.
\end{abstract}

\section{Introduction}

With the maturing of the fourth generation $(4 \mathrm{G})$ cellular networks and the emerging concepts of the fifth generation (5G) communication system [1], users are demanding access to the high-quality services anywhere and anytime [2]. In order to satisfy the increasing demands, especially for indoor application, the dense small cell deployment becomes a main direction for the traditional and next-generation communication systems $[3,4]$. Therefore, site-specific propagation models for different indoor environments with the features of low power and short distance, for example, office rooms $[5,6]$, corridors $[7,8]$, and stairs $[9,10]$, are needed to describe the channel characteristics more accurately. As a basic part of the indoor environments, stair environment plays an important role in our daily life. Moreover, stairs usually are located in the corner of buildings with inadequate wireless coverage. Detailed characterization of such an environment is, therefore, a major challenge for the successful design of the indoor coverage of future mobile communication systems.

Basic propagation mechanisms of the multifloor stairwell environment are analyzed by using ray-tracing method and then experimentally validated [9]. As the extension of the work in [9], a path loss model of multifloor stairwell with different path loss exponents in each stair section is proposed [10]. However, the effects of antenna height and the propagation environment information are rarely referred to in these models. Due to the mobility of the mobile terminals in future communication systems, the antenna height often varies with a person's posture or the condition of the mobile device (e.g., standby and in use). Additionally, there are differences in the propagation environments of stairs, for example, the existence of the cross-beams in some stairs. Hence, a path loss model including the influence of antenna height and an additional dependency of special structure in the propagation environment can more effectively describe the channel properties under indoor stair environment.

In this paper, the parameters of the novel, antennaheight-dependent path loss model are fitted using line-ofsight (LOS) and non-LOS (NLOS) data, respectively. Then, the accuracy of the model is validated by the shadowing and the fitness of measured data.

\section{Stair Structure and Measurement System}

2.1. Stair Environments. As shown in Figure 1, a typical stair environment is composed of the stair steps, railings, platforms, and so on. Additionally, some of the investigated stairs have cross-beams (the beam supporting a platform) and others do not have such beams (or the beams are very high).

In order to describe the proposed path loss model (in Section 3) more compactly, the following expressions are introduced. The part from the intermediate platform 
TABLE 1: Structure parameters of measured stair environment.

\begin{tabular}{lccccc}
\hline Section & Stair & OE & OW & T & R \\
\hline \multirow{5}{*}{ Section1 } & $N_{1}$ & 14 & 14 & 16 & 13 \\
& $R_{b}$ & 7 & 7 & 1 & 2 \\
& $h_{b}(\mathrm{~cm})$ & 273 & 267 & 334 & 294 \\
& $L(\mathrm{~cm})$ & 152 & 156 & 260 & 170 \\
& $W(\mathrm{~cm})$ & 30 & 28 & 29 & 28 \\
& $D(\mathrm{~cm})$ & 15 & 14 & 14 & 17 \\
\hline \multirow{5}{*}{ Section2 } & $N_{2}$ & 9 & 9 & 16 & 10 \\
& $R_{b}(\mathrm{~cm})$ & 198 & 197 & 334 & 230 \\
& $L(\mathrm{~cm})$ & 152 & 156 & 260 & 170 \\
& $W(\mathrm{~cm})$ & 30 & 28 & 29 & 28 \\
& $D(\mathrm{~cm})$ & 15 & 14 & 14 & 17 \\
\hline
\end{tabular}

(platform1) to the floor platform (platform2) is called the first section (S1), and the one from the platform2 to the other intermediate platform (platform3) is denoted by the second section (S2).

Four different stairs with similar structure, as in Figure 1, are measured. The different structure parameters of them are shown in Table 1 . The OE, OW, T, and R represent the east stair of an office building (17th floor), the west stair of an office building (18th floor), the stair of a teaching building (1st floor), and the stair of a residential building (2nd floor), respectively. The symbols in Figure 1 and Table 1 are explained as follows. The transmitting (Tx) and receiving $(\mathrm{Rx})$ antennas' heights are $h_{t}$ and $h_{r}$. In S1/S2, the vertical distance between the cross-beam, $\mathrm{Rx}$ antenna, and the platform1/platform 2 is denoted by $h_{b}$ and $h_{0} . L, W$, and $D$ are the length, width, and depth of a stair step, respectively. $R_{b}$ is the index of stair step under the beam, and the Rx antenna is on the Rth step. An example of $R_{b}$ and $R$ is also given in Figure 1.

2.2. Measurement System. A frequency domain measurement scheme is used [11]; the detailed description of the measurement system can be found in [12]. The main apparatus of the measurement system is an Agilent 8720ET vector network analyzer (VNA). During the measurement, the transmitting (Tx) antenna is fixed at about $2 \mathrm{~m}$ above the platform2; meanwhile, the receiving $(\mathrm{Rx})$ antenna is installed on a wooden tripod, and its height varies from $1 \mathrm{~m}$ to $1.9 \mathrm{~m}$ with the increment of $0.1 \mathrm{~m}$. The $\mathrm{Rx}$ antenna moves along the adjacent sections of the stairs step by step. Thus, according to Table 1 and the measurement scheme, the effective range of the Tx-Rx separation is about $1 \mathrm{~m}$ to $8 \mathrm{~m}$. It is envisaged to be the typical applicable range of the indoor small cell deployment (femto cell) [4]. In order to collect more data, at each stair step, five different positions (grids) are measured. Both of $\mathrm{Tx}$ and $\mathrm{Rx}$ antennas are omnidirectional antennas with gains of $3 \mathrm{dBi}$ and are connected to the two ports of the VNA via a $10 \mathrm{~m}$ and $2.5 \mathrm{~m}$ coaxial cable (the cable attenuation is $0.6 \mathrm{~dB} / \mathrm{m}$ ), respectively. The VNA sweeps from $2.5 \mathrm{GHz}$ to $2.69 \mathrm{GHz}$ with the power of $10 \mathrm{dBm}$. During each sweep, by capturing the transmission coefficient $S_{21}, 201$ complex frequency responses are recorded on a laptop via GPIB

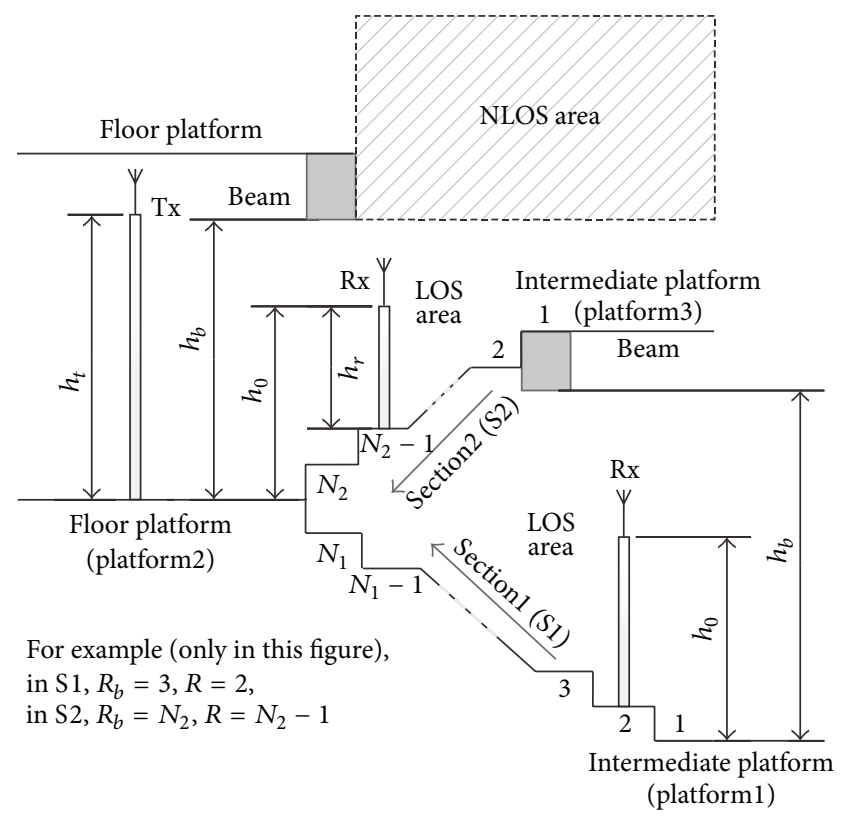

FIgURE 1: Typical stair environment.

interface. In order to eliminate the influence of the noise, 5 groups of frequency responses are measured at each stair step and $\mathrm{Rx}$ antenna height.

\section{Antenna-Height-Dependent Path Loss Model}

Conventionally, the path loss is modeled as a log-distance function with a log normally distributed shadowing [13]. To characterize the path loss under indoor stair environment more accurately, the receiving antenna height and detailed information about the stair (such as the beam) should be introduced into traditional path loss model.

3.1. Influence of the Cross-Beam. The existence of cross-beam with low height in some stairs may cause the obstruction of the LOS path (direct path) between the Tx and Rx antennas. Such obstruction may lead to differences in the path loss model. Therefore, according to the height and position of $\mathrm{Rx}$ antenna and the cross-beam, all the measurement points can be categorized into LOS and NLOS ones, using (1). As an example, in Figure 1, the beam in S1 is high; therefore, there is no obstruction between Tx and Rx antennas; meanwhile, if the Rx antenna falls within the shadowing area (denoted by NLOS area in Figure 1), the LOS path will be blocked by the beam:

$$
\begin{array}{ll}
\text { NLOS } & R<R_{b}, h_{0}>h_{b}, \\
\text { LOS } & \text { otherwise. }
\end{array}
$$

Figure 2 shows the scatter plots and corresponding fit curves of the measured path loss. It is seen that a fixed difference exists between the LOS and NLOS fitted curves. Therefore, a constant can be defined to describe the influence 
of the cross-beam. It is zero if the $\mathrm{Rx}$ antenna is located in LOS area and is a positive value for NLOS point as follows:

$$
C= \begin{cases}0, & \text { LOS } \\ c, & \text { NLOS }\end{cases}
$$

3.2. Expression of the Antenna Height Gain Factor. In order to describe the influence of the antenna height on the path loss, an Rx antenna height gain factor $G\left(h_{r}\right)$ is introduced. By subtracting the reference path loss, the cross-beam attenuation, and the path loss caused by distance, the antenna gain factor, $G\left(h_{r}\right)$, is extracted. Using least square (LS) method, some functions are used to fit the measure data. As examples, the fitting mean square error (MSE) of the logarithmic function $G\left(h_{r}\right)=A \log _{10}\left(h_{r} / B\right)$, the power function $G\left(h_{r}\right)=A h_{r}^{B}$, the exponential function $G\left(h_{r}\right)=A \exp \left(B h_{r}\right)$, and linear function $G\left(h_{r}\right)=A h_{r}+B$ are 11.3, 13.6, 13.9, and 11.4, respectively. It is seen that the logarithmic function fits the measured data best. Therefore, the $\mathrm{Rx}$ antenna height gain factor is described using (3), which is similar to the antenna height gain factor of Okumura model [13]. However, the Okumura model is generally used for outdoor propagation prediction in urban area. Therefore, the parameters of $\mathrm{Rx}$ antenna height gain factors, $A$ and $B$, should be recalculated under indoor propagation environment:

$$
G\left(h_{r}\right)=A \log _{10}\left(\frac{h_{r}}{B}\right) .
$$

Figure 3 plots the path loss gain versus $\mathrm{Rx}$ antenna height. The logarithmic function can be used to describe the effect of the $\mathrm{Rx}$ antenna height on the path loss. $G\left(h_{r}\right)$ is an increasing function for LOS data; meanwhile, it is a decreasing one for NLOS data. The causes for such variation trends are explained in Section 4.

3.3. Proposed Path Loss Model. Based on the above analysis, the influence of the cross-beam and the antenna height should be considered, and a novelpath loss model under stair environment could be described as

$$
\mathrm{PL}\left(d, h_{r}\right)=\mathrm{PL}\left(d_{0}\right)+10 n \log _{10}\left(\frac{d}{d_{0}}\right)+G\left(h_{r}\right)+C+X_{\sigma},
$$

where $d$ is the separation between $\mathrm{Tx}$ and $\mathrm{Rx}$ antennas, $\operatorname{PL}\left(d, h_{r}\right)$ is the predicted path loss, $n$ is the path loss exponent, $\mathrm{PL}\left(d_{0}\right)$ is the reference path loss calculated using the free space path loss formula (usually $d_{0}=1 \mathrm{~m}$ under indoor environment), $C$ is the cross-beam attenuation factor as (2), $G\left(h_{r}\right)$ is the Rx antenna gain factor described by (3), and $X_{\sigma}$ is a normal random variable in $\mathrm{dB}$ with zero mean $(\mu)$ and standard deviation of $\sigma \mathrm{dB}$.

\section{Parameters Extraction and Validation}

The variation caused by the stair structures will lead to different parameters in the path loss model, such as $n, A, B$, and $C$. However, the proposed model is a statistical model

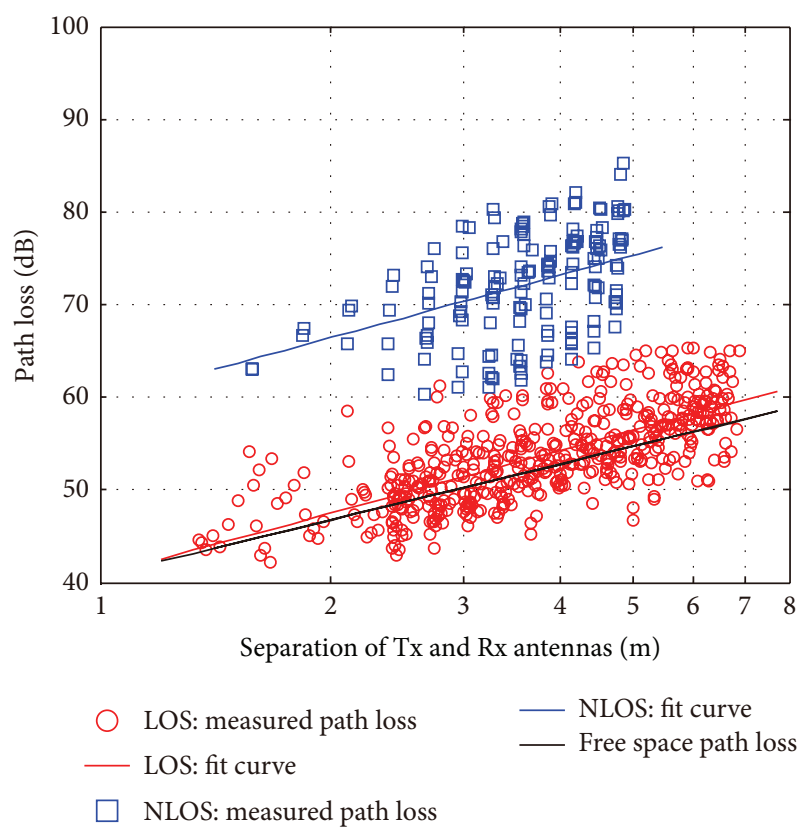

FIGURE 2: Scatter plots of the path loss, the corresponding fit curves, and free space path loss.

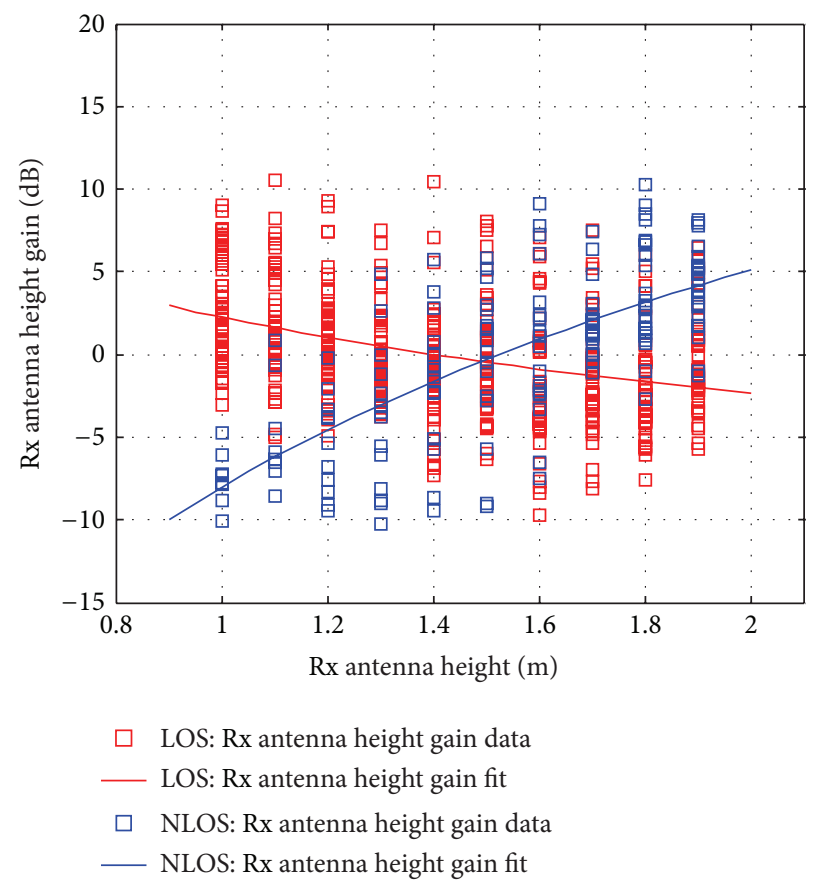

Figure 3: Fit of the Rx antenna height gain factor.

based on intensive measurements. In order to get a more general path loss model, all the measured data (divided into LOS and NLOS data) of different stairs are used to fit the model (4). Four sets of extracted path loss data in the different floors of the office, teaching, and residential buildings are used, and the population of used data is 25250 (including 101 stair steps, 5 grids, 5 measured groups, and 10 antenna heights). The parameters $n, A, B$, and $C$ are regressed using 
TABle 2: Parameters of the proposed path loss model.

\begin{tabular}{lcc}
\hline & LOS & NLOS \\
\hline $\mathrm{PL}\left(d_{0}\right)(\mathrm{dB})$ & 40.7 & 40.7 \\
$n$ & 2.25 & 2.25 \\
$A$ & -15.3 & 43.7 \\
$B$ & 1.4 & 1.5 \\
$C(\mathrm{~dB})$ & 0 & 18.9 \\
$\mu(\mathrm{dB})$ & 0 & 0 \\
$\sigma(\mathrm{dB})$ & 3.3 & 3.5 \\
\hline
\end{tabular}

the least square (LS) method. The $\mu$ and $\sigma$ are fitted by the maximum likelihood estimation (MLE). All the parameters of the proposed path loss model are summarized in Table 2. Notably, although the parameters listed in Table 2 are related to specific building materials in which the measurements are carried out, the proposed model (4) can be applied to other stairs with similar structure.

As shown in Figure 2, the existence of the beam causes about $19 \mathrm{~dB}$ attenuation in path loss. This attenuation is large compared with overall path loss value. For comparison, the free space path loss is also plotted in Figure 2. It is observed that the measured path loss deviates from the free space path loss curve, especially for the NLOS data.

In Figure 3, it is seen that, in LOS area, the antenna height gain factor is a decreasing function of the $\mathrm{Rx}$ antenna height. This result is consistent with the results presented in [14]. However, the decrease becomes slower at higher antenna height. This can be explained as follows. The attenuation caused by the railings, walls, and steps is large at low antenna height. With the increase of the antenna height, the influence of the attenuation caused by these surroundings becomes smaller. Meanwhile, the attenuation caused by the ceiling becomes larger. Therefore, the antenna height gain factor represents such variation tendency. In NLOS area, the higher the antenna height is, the more the radiation pattern of the Tx and Rx antennas is obstructed by the beam, leading to the antenna height gain factor being an increasing function.

The shadowing represents that the measured path loss varies statistically around the path loss fit curve [15]. Figure 4 shows the empirical and fit cumulative distribution functions (CDFs) of the shadowing. The distributions of the shadowing in LOS and NLOS areas are almost the same (3.3 for LOS data, 3.5 for NLOS data). That means the correction factors ( $C$ and $\left.G\left(h_{r}\right)\right)$ in the proposed model can precisely characterize the overall path loss for both of LOS and NLOS points.

In order to validate the accuracy of the proposed path loss model, shadowing effect of the path loss is considered. The traditional log-distance path loss model only takes the Tx$\mathrm{Rx}$ distance into consideration, and not any of the physical features of the propagation environment is included. Due to the above reason, several measurements of the same Tx-Rx antennas separation will have widely varying path loss values. With more site-specific information being given, the value of standard deviation becomes lower. Therefore, the precision of a path loss model can be evaluated by the standard deviation of the shadowing effect [16].

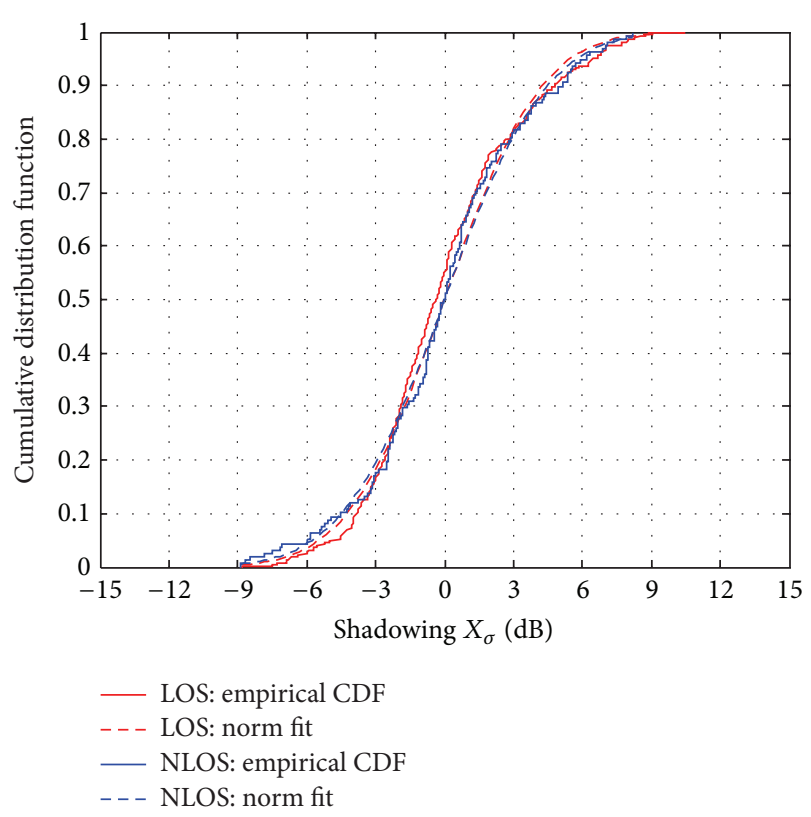

FIGURE 4: CDFs of the shadowing of the proposed model (LOS and NLOS points).

For comparison, the parameters of the log-distance model are calculated using the measured data. The path loss exponent of log-distance model is 3.0. The CDFs of the shadowing are plotted in Figure 5. The $\sigma$ of the proposed model and log-distance model is 3.4 and 9.1, respectively. It is observed that the proposed model has a smaller $\sigma$ compared with log-distance path loss model. This is because the model in (4) contains more site-specific information, that is, information about antenna height and the attenuation of the beam.

The measured path loss, the proposed model, and logdistance model surfaces are shown in Figure 6. It is seen that the proposed model fits to the measured data well. Both of the reduction in standard deviation of the shadowing and the high degree of agreement with the measured data prove that the proposed model is accurate and effective.

\section{Conclusion}

The path loss model with receiving antenna height dependency under indoor stair environment is proposed based on intensive measurements. The parameters of the model are extracted using LS method and MLE. In the proposed model, the antenna height gain factor is a decreasing function in LOS condition and an increasing function in NLOS condition. It is also found that the beam can cause a large attenuation of the path loss. The stair structure under investigation is very common. Thus, the proposed model can be applied to predict the signal level of different steps and different antenna height with such stair structure and provides the criterion of cell planning of indoor environment. 


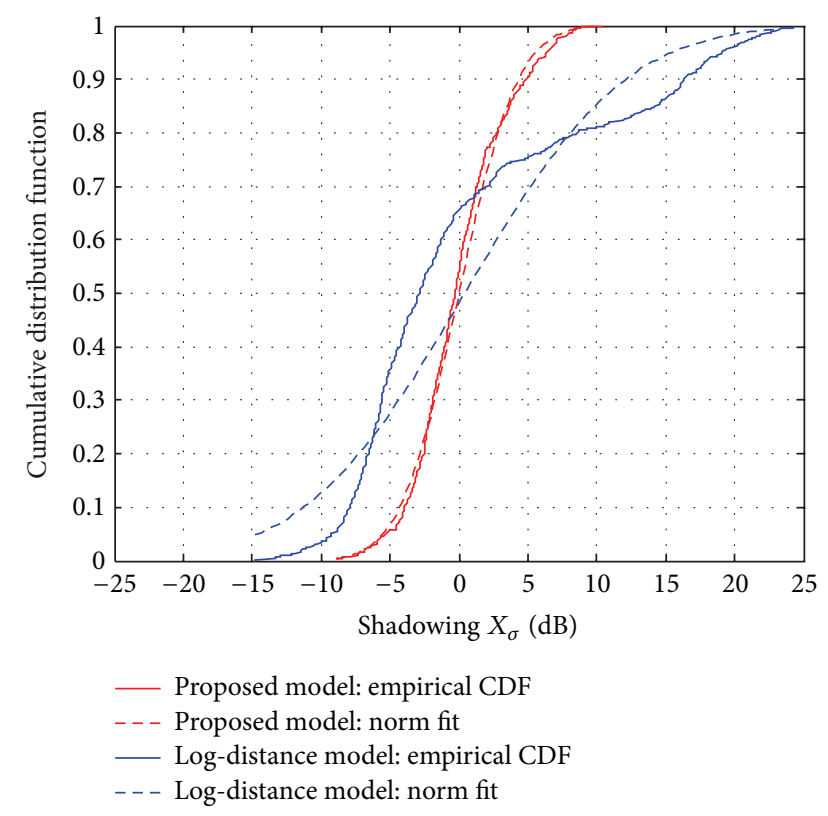

Figure 5: CDFs of overall shadowing of the proposed model and log-distance model.

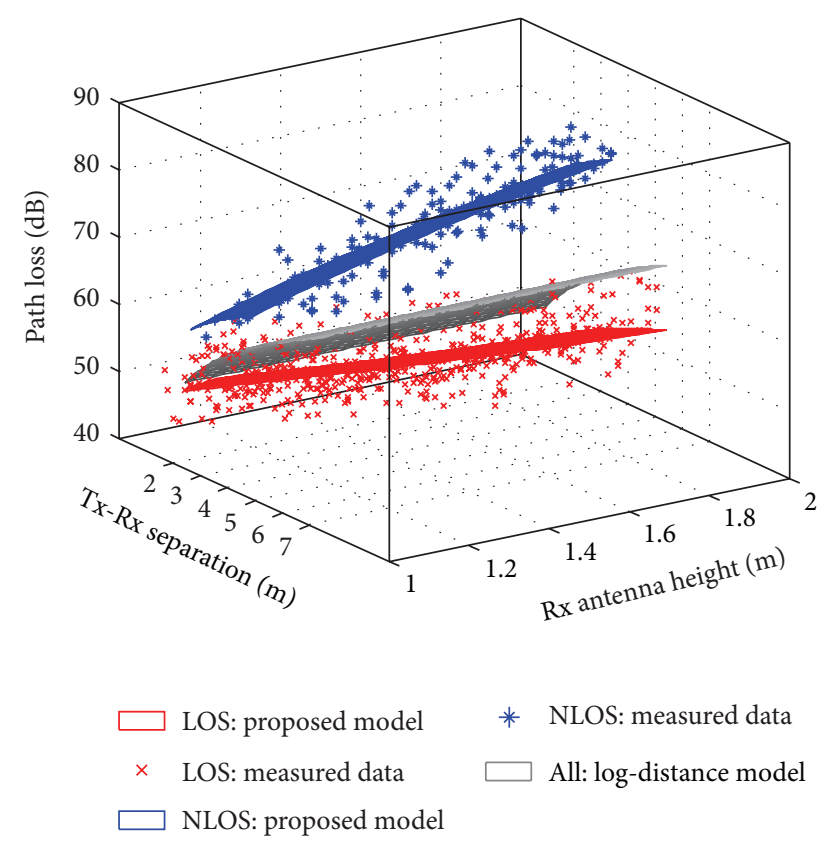

FIGURE 6: Scatter plots of measured data and corresponding fit surfaces of the proposed model and the log-distance model.

\section{Conflict of Interests}

The authors declare that there is no conflict of interests regarding the publication of this paper.

\section{Acknowledgments}

This work is supported by the National 863 Program under Grant no. 2014AA01A705 and National Nature Science Foundation Council (NSFC) Proposal under Grant no. 61271237. The authors are also indebted to Mr. Jing Liu and Mr. Bo Yang, China Telecommunication Technology Laboratory (CTTL), for their help and assistance in the channel measurement.

\section{References}

[1] I. Chih-Lin, C. Rowell, S. Han, Z. Xu, G. Li, and Z. Pan, “Toward green and soft: a $5 \mathrm{G}$ perspective," IEEE Communications Magazine, vol. 52, no. 2, pp. 66-73, 2014.

[2] I. Akyildiz, S. Mohanty, and J. Xie, "A ubiquitous mobile communication architecture for next-generation heterogeneous wireless systems," IEEE Communications Magazine, vol. 43, no. 6, pp. S29-S36, 2005.

[3] C. Gee-Kung, L. Cheng, and Z. Liang, "Architecture and applications of a versatile small-cell, multi-service cloud radio access network using radio-over-fiber technologies," in Proceedings of the IEEE International Conference on Communications Workshops, pp. 879-883, June 2013.

[4] V. Chandrasekhar, J. G. Andrews, and A. Gatherer, "Femtocell networks: a survey," IEEE Communications Magazine, vol. 46, no. 9, pp. 59-67, 2008.

[5] D. Xu, J. Zhang, X. Gao, P. Zhang, and Y. Wu, "Indoor office propagation measurements and path loss models at $5.25 \mathrm{GHz}$," in Proceedings of the IEEE 66th Vehicular Technology Conference (VTC '07), pp. 844-848, Baltimore, Md, USA, October 2007.

[6] J. Choi, N.-G. Kang, Y.-S. Sung, J.-S. Kang, and S.-C. Kim, "Frequency-dependent UWB channel characteristics in office environments," IEEE Transactions on Vehicular Technology, vol. 58, no. 7, pp. 3102-3111, 2009.

[7] X. Zhao, S. Geng, and B. M. Coulibaly, "Path-loss model including LOS-NLOS transition regions for indoor corridors at $5 \mathrm{GHz}$ [wireless corner]," IEEE Antennas and Propagation Magazine, vol. 55, no. 3, pp. 217-223, 2013.

[8] T. R. Rao, D. Murugesan, S. Ramesh, and V. A. Labay, "Radio channel characteristics in an indoor corridor environment at $60 \mathrm{GHz}$ for wireless networks," in Proceedings of the IEEE 5th International Conference on Advanced Networks and Telecommunication Systems (ANTS '11), pp. 1-5, IEEE, Bangalore, India, December 2011.

[9] S. Y. Lim, Z. Yun, J. Baker, N. Celik, H. S. Youn, and M. Iskander, "Propagation modeling and measurement for a multifloor stairwell," IEEE Antennas and Wireless Propagation Letters, vol. 8, pp. 583-586, 2009.

[10] S. Y. Lim, Z. Q. Yun, and M. F. Iskander, "Radio propagation modeling in indoor stairwell: a K-means clustering approach," in Proceedings of the IEEE Antennas and Propagation Society International Symposium, pp. 1-2, Chicago, Ill, USA, 2012.

[11] H. Zaghloul, G. Morrison, and M. Fattouche, "Frequency response and path loss measurements of indoor channel," Electronics Letters, vol. 27, no. 12, pp. 1021-1022, 1991.

[12] Y. Wang, W.-J. Lu, and H.-B. Zhu, "Propagation characteristics of the LTE indoor radio channel with persons at $2.6 \mathrm{GHz}$," IEEE Antennas and Wireless Propagation Letters, vol. 12, pp. 991-994, 2013.

[13] T. S. Rappaport, Wireless Communications: Principles and Practice, vol. 2, Prentice Hall PTR, Upper Saddle River, NJ, USA, 1996. 
[14] U. Shukla, H. Volos, and R. Buehrer, "On the effect of antenna height on the characterization of the indoor UWB channel," in Proceedings of the IEEE Global Telecommunications Conference, pp. 1-5, November 2008.

[15] D. Cassioli, M. Z. Win, and A. F. Molisch, "The ultra-wide bandwidth indoor channel: from statistical model to simulations," IEEE Journal on Selected Areas in Communications, vol. 20, no. 6, pp. 1247-1257, 2002.

[16] J. Andersen, T. Rappaport, and S. Yoshida, "Propagation measurements and models for wireless communications channels," IEEE Communications Magazine, vol. 33, no. 1, pp. 42-49, 1995. 

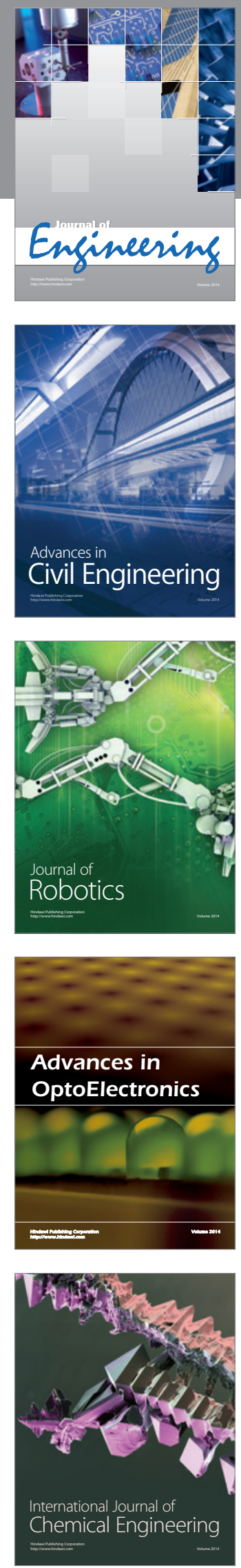

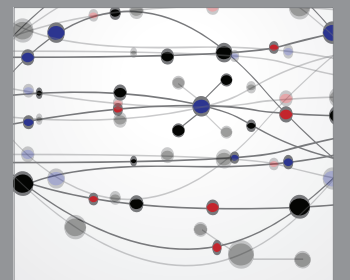

The Scientific World Journal
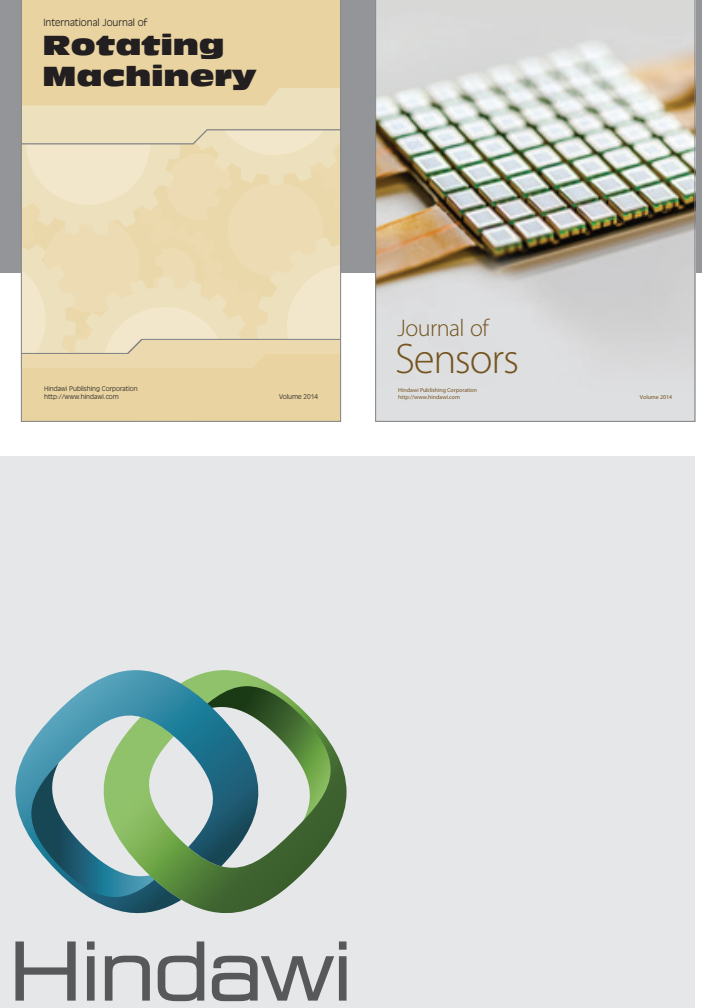

Submit your manuscripts at http://www.hindawi.com
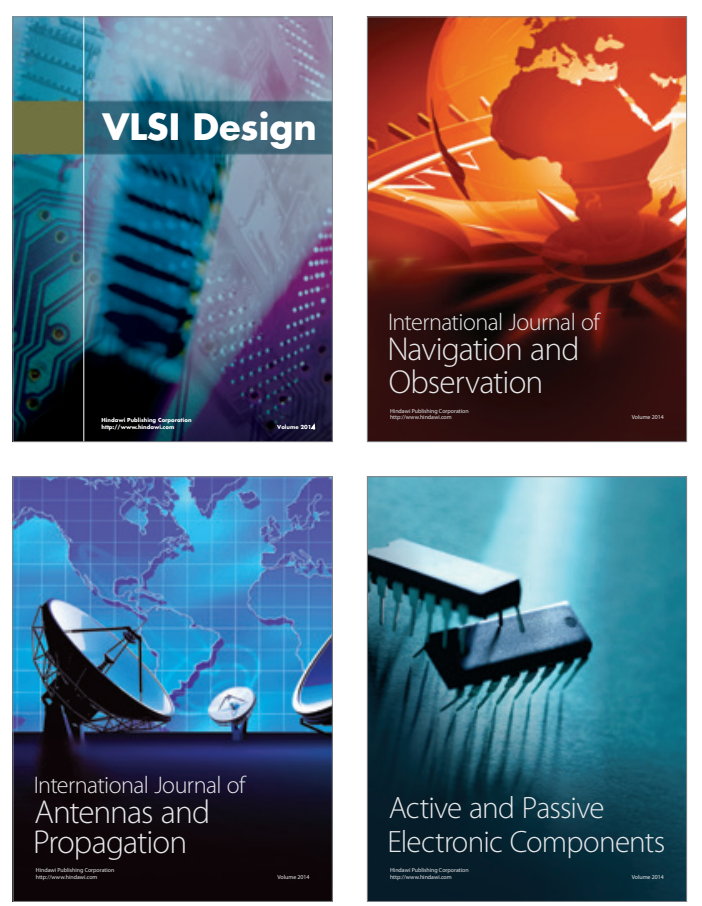
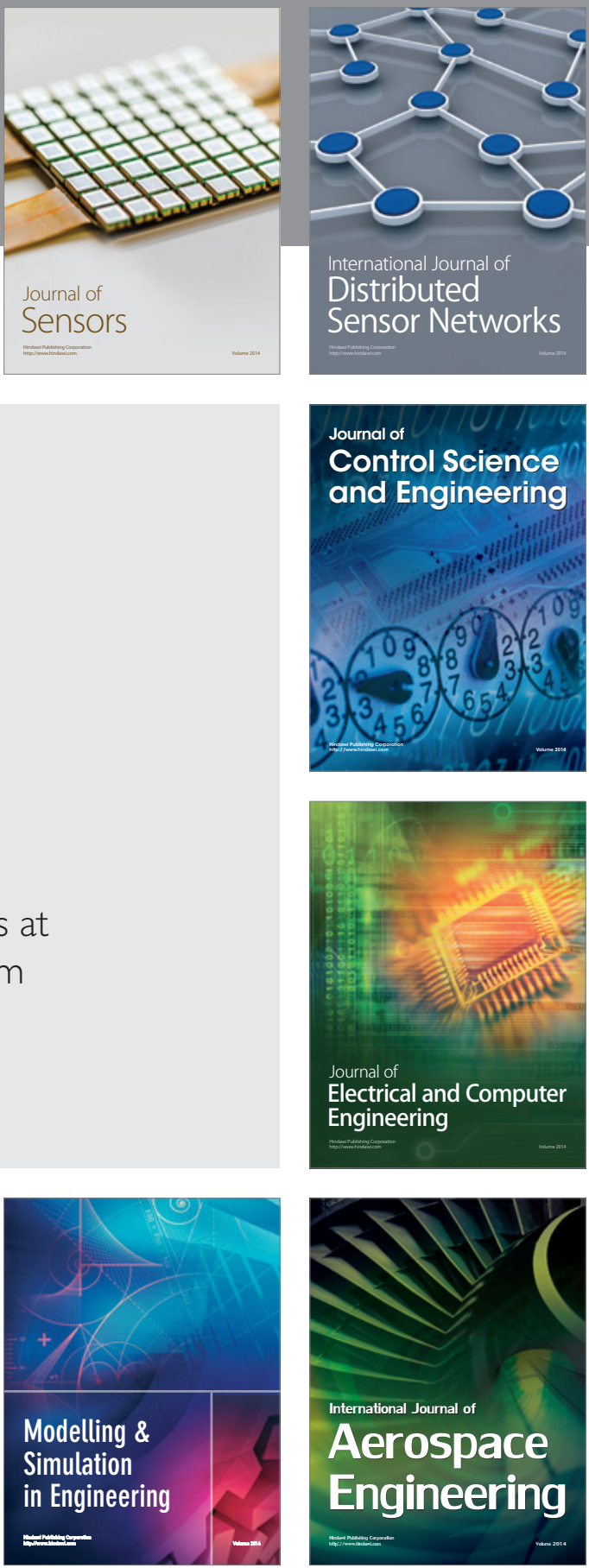

Journal of

Control Science

and Engineering
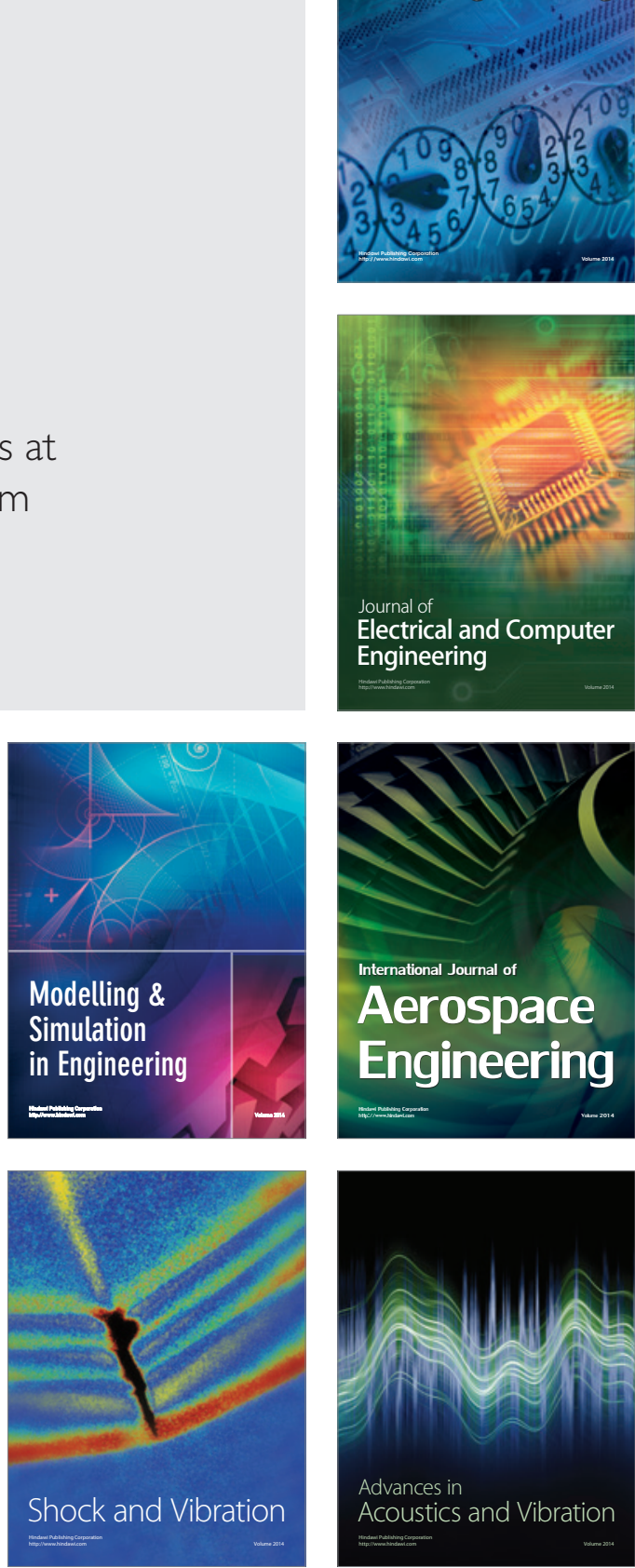